\title{
Wall pressure measurements of flooding in vertical countercurrent annular air-water flow
}

\author{
I. Choutapalli ${ }^{\mathrm{a}, *}$, K. Vierow ${ }^{\mathrm{b}}$ \\ a Department of Mechanical Engineering, University of Texas - Pan American, Edinburg, TX 78539, USA \\ ${ }^{\mathrm{b}}$ Department of Nuclear Engineering, Texas AEM University, College Station, TX 77843, USA
}

\section{A R T I C L E I N F O}

\section{Article history:}

Received 6 August 2009

Received in revised form 17 May 2010

Accepted 4 June 2010

Available online $\mathrm{xxx}$

\begin{abstract}
A B S T R A C T
An experimental study of flooding in countercurrent air-water annular flow in a large diameter vertical tube using wall pressure measurements is described in this paper. Axial pressure profiles along the length of the test section were measured up to and after flooding using fast response pressure transducers for three representative liquid flow rates representing a wide range of liquid Reynolds numbers $\left(\operatorname{Re}_{\mathrm{L}}=4 \Gamma / \mu\right.$; $\Gamma$ is the liquid mass flow rate per unit perimeter; $\mu$ is the dynamic viscosity) from 3341 to 19,048 . The results show that flooding in large diameter tubes cannot be initiated near the air outlet and is only initiated near the air inlet. Fourier analysis of the wall pressure measurements shows that up to the point of flooding, there is no dominant wave frequency but rather a band of frequencies encompassing both the low frequency and the broad band that are responsible for flooding. The data indicates that flooding in large diameter vertical tubes may be caused by the constructive superposition of a plurality of waves rather than the action of a single large-amplitude wave.
\end{abstract}

(C) 2010 Elsevier B.V. All rights reserved.

\section{Introduction}

The condition of countercurrent flow limitation (CCFL) or flooding represents the stable limit of a countercurrent gas-liquid annular flow. The annular flow is comprised of a gas core flow upward surrounded by a falling liquid film. At low gas flow rates, the liquid film can simply fall under gravity creating a countercurrent flow. However, higher gas flow rates have enough momentum to reverse the liquid flow direction and carry it upwards giving rise to a cocurrent flow. The point of transition from a countercurrent flow to a partial or complete flow reversal is known as flooding and has been the subject of investigation for a number of decades. Annular flows are of great importance for the operation and efficiency of different process equipment such as nuclear reactors, reflux condensers, desiccant cooling systems, wetted wall columns, etc. For example, in a hypothetical accident scenario in a nuclear reactor, the Emergency Core Cooling System injects water into the reactor pressure vessel. Should upward steam flow prevent sufficient cooling, safe operation of the reactor may be compromised. It is therefore of utmost importance that this phenomenon is thoroughly understood to enhance the thermal hydraulic safety of future nuclear reactors, and hence promote better design of the coolant piping and flow paths.

\footnotetext{
* Corresponding author. Tel.: +1 9563185220.

E-mail addresses: choutapalliim@utpa.edu, isaac.choutapalli@gmail.com (I. Choutapalli).
}

The growth and propagation of interfacial waves have been widely considered as determinants for the conditions of flooding in countercurrent two-phase flows. One of the direct methods of studying the effects of interfacial disturbances is by investigating the wall pressure fluctuations. Interfacial disturbances generate velocity fluctuations that give rise to hydrodynamic pressure fluctuations. The pressure disturbances that arise as a result of these fluctuations propagate through the liquid film in multiple directions depending on the shape of the interface and the presence of liquid droplets. The magnitude and frequency of the pressure disturbances can be captured using wall pressure measurements. The wall pressure measurements would contain both the local and global hydrodynamic nature of the two-phase flow. In addition, they also contain the convected pressure due to any entrained liquid droplets present in the flow (Samways et al., 1997). In this paper, detailed measurements of wall pressure along with relevant flow visualization will be presented to further the understanding of flooding in countercurrent vertical air-water annular flows.

The air-water countercurrent flow is highly turbulent and stochastic in nature. Even in the absence of countercurrent air flow, a falling annular liquid film is characterized by surface waves and is inherently unstable. Any small perturbation can lead to a possible surface wave amplification. The introduction of countercurrent air flow can be thought of as introducing a large-scale perturbation resulting in pressure-velocity fluctuations. The magnitude of pressure-velocity fluctuations will determine the extent of momentum exchange between air and water and consequently lead to flooding. Despite a number of years of research, many 
aspects of flooding still remain unclear. One of the fundamental questions this study seeks to answer is the role of wave frequencies generated due to pressure fluctuations and wall pressure variation in initiating flooding. A study of wall pressure fluctuations at different locations and flow conditions will help answer these fundamental questions related to flooding in countercurrent air-water flow in large diameter vertical tubes.Wall pressure measurements have been used in the study of two-phase flows for a wide range of applications and in different flow geometries. Semenov (1959) was one of the first to carry out unsteady wall pressure fluctuations in air-water slug flows. The unsteady wall pressure generated due to the motion of the slugs was studied and it was found that the observed dominant frequencies were relatively low. Maximum amplitude of the pressure fluctuations was observed when the void fraction was about 0.90. Hubbard and Dukler (1966) carried out measurements of wall pressure fluctuations in horizontal gas-liquid flow and presented the results using Fourier analysis. Nishikawa et al. (1969) carried out wall pressure measurements in a $26 \mathrm{~mm}$ tube using static strain gauge transducers. The main goal behind their study was to identify different flow regimes based on the probability density function. They identified annular and bubbly flow regimes by comparing the probability density function to a Gaussian distribution. Davis (1973) studied the unsteady pressure fluctuations in a low velocity $(5 \mathrm{~m} / \mathrm{s})$ turbulent flow of a gas-liquid mixture of freon flowing vertically upwards in a circular pipe. The flow was mainly bubbly and the propagation of the pressure disturbances due to the presence of the bubbles was presented. Tutu (1982) carried out wall pressure measurements on a twophase flow loop with an internal tube diameter of $52.2 \mathrm{~mm}$. The piezoresistive transducers were flush mounted with the tube inner surface for accurate measurements. He also recorded static wall pressure measurements both upstream and downstream of these transducers to obtain the differential pressure. Based on the probability density function, skewness and flatness of the differential pressure measurements, Tutu (1982) claimed that different flow regimes could be identified. Matsui (1984) also used flush mounted piezoresistive transducers to study the differential pressure variation in the flow of nitrogen and water in a tube of internal diameter $22 \mathrm{~mm}$. He used cross-correlation techniques to obtain the phase lag and the position of the peak was assumed to correspond to the dispersed phase velocity and also calculated the corresponding velocity of the rising gas bubble.

Sami and Lakis (1986) studied the spectral characteristics of wall pressure in a closed two-phase flow loop system. From their study, the frequency spectra of the wall pressure fluctuations showed very low amplitudes and the convection of pressure disturbances was found to increase with the two-phase flow mixing ratio. Drahos et al. (1996) and Cai et al. (1996) used pressure measurements to study the turbulent nature of horizontal air-water flow. Langford et al. (1998) carried out wall pressure measurements in vertical two-phase flows and used the probability density function to identify different flow regimes as well as relate turbulence measurements to superficial liquid and gas velocities. Samways et al. (1997) used wall pressure fluctuation measurements in bubbly flows and found that the bubble phase created low fluctuating pressures. McClusky et al. (2002) used wall pressure measurements of vertical gas-liquid flows and performed a wavelet analysis to determine the time variation of the frequency content in the pressure signals.

The study of unsteady wall pressure fluctuations in relation to flooding in large diameter vertical tubes has, however, not been investigated. It is therefore the aim of the present investigation to study flooding in light of unsteady wall pressure measurements. The experimental work presented in this paper will use wall pressure measurements of vertical countercurrent annular air-water flow to demonstrate that the frequency content in the pressure signals up to the point of flooding is a continuous spectrum and not a single dominant frequency. A description of these findings is presented in this manuscript.

\section{Experimental apparatus and measurement techniques}

\subsection{Experimental setup}

Fig. 1 shows the schematic of the experimental setup. It consists of a test section $1800 \mathrm{~mm}$ long, $76.2 \mathrm{~mm}$ inner diameter and constructed from optically transparent acrylic (Solmos, 2008). Two cylindrical plenum chambers, also constructed from optically transparent acrylic are attached to the test section, one at the top and another at the bottom. The upper plenum chamber assembly is designed to serve a twofold purpose of ensuring annular liquid flow into the test section as well as serve as an outlet for air and any entrained liquid leaving the test section. It is a cast acrylic tube of $127.0 \mathrm{~mm}$ inner diameter and $152.4 \mathrm{~mm}$ long. Into this tube, four $25.4 \mathrm{~mm}$ holes in diametrically opposite directions have been drilled to allow water to enter the plenum. The plenum is attached to the test section by means of flanges located in such a way that the upper $152.4 \mathrm{~mm}$ length of the test section passes through the plenum chamber concetrically. This arrangement creates a uniform circumferential gap of $19.05 \mathrm{~mm}$ between the inner surface of the plenum and the outer surface of the test section contained inside the plenum chamber.

The upper part of the test section contained inside the plenum chamber has twelve $6.35 \mathrm{~mm}$ circular holes spaced uniformly along the circumference and on the same axial plane (section A-A of Fig. 1). This axial plane is located at the longitudinal midsection of the upper plenum. The water entering the upper plenum penetrates through these holes present around the test section circumference and impinges on an inner tube ( shown in section A-A). This arrangement forces the water to flow down into the test section as an annular flow. The inner tube extends $25.4 \mathrm{~mm}$ below the upper plenum chamber and $100.0 \mathrm{~mm}$ above the plenum. It also acts as an outlet for the air and entrained water leaving the test section.

Air is supplied to the test section from a blower (Republican blower model 11RB-210). The air from the blower is routed through a long straight tube of $38.1 \mathrm{~mm}$ inner diameter (length/diameter ratio of 30) to ensure a fully developed flow. A flexible hose of the same inner diameter connects this straight tube to the air inlet of the test section. The air inlet of the test section consists of a straight tube $762 \mathrm{~mm}$ long and $38.1 \mathrm{~mm}$ inner diameter passing concentrically through the center of the lower plenum chamber and extending $50.8 \mathrm{~mm}$ into the test section as shown in Fig. 1. The lower plenum chamber is also designed to drain the water from the test section into the water collection chamber as shown in the figure.

\subsection{Instrumentation}

The water flow rate into the test section is measured using a magnetic flow meter (Yamatake, MagneW3000 Plus) located sufficiently upstream of the test section water inlet as shown in Fig. 1. The air velocity is measured with a pitot-static tube located at the downstream end of the straight tube connected to the blower. The total and static pressure ports of the pitot tube are connected to a Honeywell differential pressure transducer (ST3000 SMART pressure transmitter) calibrated for the range of pressures encountered during the operation of the blower. Two Kulite pressure transducers (model \# XT-190-5D) are used to acquire high-frequency pressure data. The transducers are flush mounted on the inner surface of the test section. Five pressure ports are located on the test section wall at a distance of $0.20,0.58,0.96,1.34$ and $1.54 \mathrm{~m}$ from the exit plane 


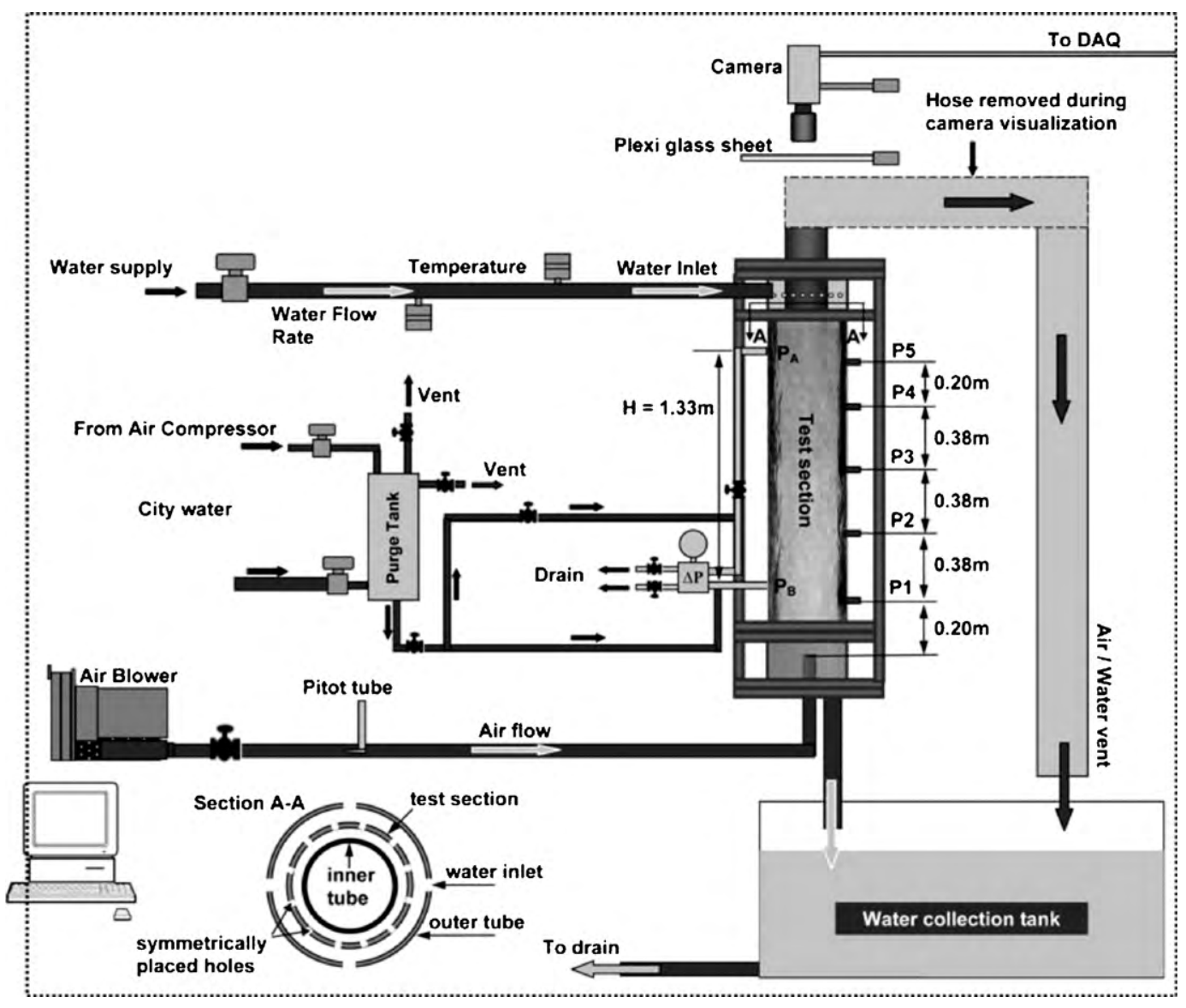

Fig. 1. Schematic of the experimental setup.

of the air inlet (Fig. 1). The pressure data was collected at these pressure ports along the length of the test section by relocating the transducers for a given flow condition. The data is sampled at a rate of $5000 \mathrm{~Hz}$. The mean pressure drop across two other ports located diametrically opposite to the Kulite pressure ports and separated by a distance of $1.33 \mathrm{~m}$ (Fig. 1) is also measured separately using a Honeywell differential pressure transducer. The uncertainty in all the wall pressure measurements is $\pm 0.05 \mathrm{kN} / \mathrm{m}^{2}$. All the data is acquired through a NI PCI-MIO-16E data acquisition card through a LabView program with an SCXI-1000 module serving as a chasis.

Flow visualization was conducted with a high speed camera positioned at the center of the test section and approximately $150 \mathrm{~mm}$ above the water inlet. The images are recorded by a CMOS camera (IDT XS-4) with a resolution $512(\mathrm{H}) \times 512(\mathrm{~V})$ pixels of a size of $16 \mu \mathrm{m} \times 16 \mu \mathrm{m}$ using the IDT MotionPro software. The camera was fitted with a $55 \mathrm{~mm}(f / 2.8)$ Nikon lens. The resulting image area covered by the camera for these set of experiments is $1.5 D(\mathrm{H}) \times 1.5 D(\mathrm{~V})$ or approximately $80 \mathrm{~mm} \times 80 \mathrm{~mm}$. Here, $D$ is the inner diameter of the test section.

\section{Results and discussion}

\subsection{Mean wall pressure gradient}

The pressure gradient in annular air-water flow arises from the contribution of (i) hydrostatic head, (ii) flow acceleration and (iii) friction. The overall pressure gradient from the three contributions can be expressed as

$\frac{d P}{d z}=\left(\frac{d P}{d z}\right)_{\text {static }}+\left(\frac{d P}{d z}\right)_{\text {accl }}+\left(\frac{d P}{d z}\right)_{\text {friction }}$
The pressure gradient due to the hydrostatic head is defined as

$\left(\frac{d P}{d z}\right)_{\text {static }}=\rho g H$

where $\rho$ is the fluid density and $H$ is the axial distance between two points in the flow. If the air-water annular flow is not fully developed, the pressure gradient due to flow acceleration has to be taken into account and is given by

$\left(\frac{d P}{d z}\right)_{a c c l}=\frac{d}{d z}\left(\frac{1}{A} \int_{A} \rho u^{2} d A\right)$

where $u$ is the effective fluid velocity. This term includes interfacial shear. In the present experimental setup, the $L / D$ (length to diameter ratio) of the test section is 23.6. An $L / D$ ratio of at least 50 is required to ensure a fully developed annular flow in circular tubes. Therefore, in these experiments, flow acceleration may have a significant contribution to the overall pressure gradient. The last term in Eq. (1), i.e. the frictional pressure drop, arises as a result of the wall shear stress exerted by the tube wall on the fluid. If $\tau_{\mathrm{w}}$ is the wall shear stress, the frictional pressure gradient can be expressed as

$\left(\frac{d P}{d z}\right)_{\text {friction }}=\frac{4 \tau_{\mathrm{w}}}{D}$

The mean wall pressure measurements described in the subsequent sections contain the contributions from all the three terms mentioned in Eq. (1) and also from any convected pressure disturbance due to the presence of liquid droplets in the flow.

In order to measure the mean wall pressure gradient, the mean wall pressure differential, $\Delta P$ across two points was measured. It 


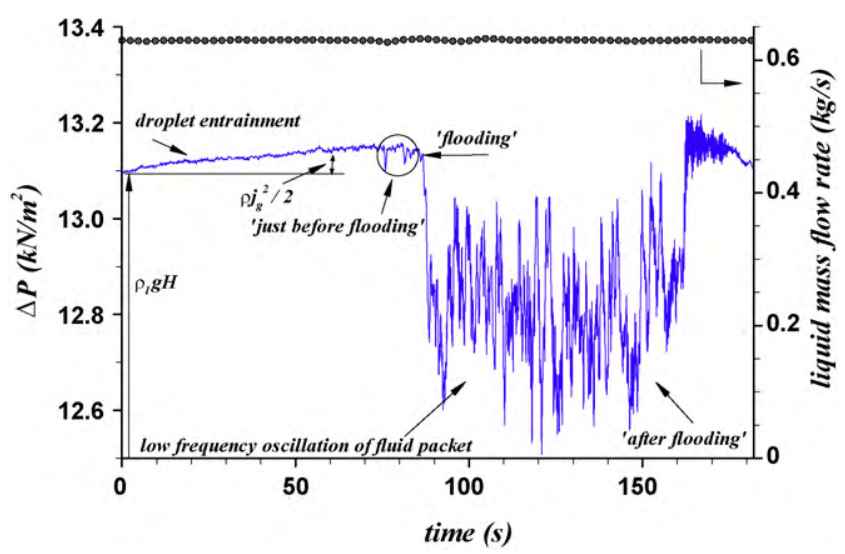

Fig. 2. Variation of $\Delta P$ with time; liquid flow rate $=0.63 \mathrm{~kg} / \mathrm{s}$.

can be expressed as

$\Delta P=P_{A}-P_{B}$

where $P_{A}$ and $P_{B}$ are the top and bottom mean absolute pressures respectively (Fig. 1). $\Delta P$ is directly measured using the Honeywell differential pressure transducer. The $\Delta P$ for a liquid flow rate of $0.63 \mathrm{~kg} / \mathrm{s}$ is shown in Fig. 2. The data is also used to quantitatively identify the time at which flooding is initiated. The superficial air velocity $j_{g}$ ( $j_{g}=$ mass flow rate of air/test-section cross-sectional area), is increased incrementally until a large change in the magnitude of the pressure drop, $\Delta P$, is observed as shown in Fig. 2 . The time at which this large change in $\Delta P$ occurs is taken to be the onset of flooding. For the water flow rate under consideration, the initial change in $\Delta P$ at the onset of flooding is about $0.4 \mathrm{kN} / \mathrm{m}^{2}$. The definition of flooding has varied widely in the literature. But it is generally agreed upon that flooding in a countercurrent flow can be determined in several ways (Bankoff and Lee, 1986). Flooding can be manifested as a large change in $\Delta P$ as observed in Fig. 2 or by the appearance of large-amplitude waves on the falling water film or droplets of liquid entrained out of the test section due to the countercurrent flow of gas. However, in the present study, the appearance of waves or droplet entrainment did not result in any significant change in the magnitude of $\Delta P$. Therefore, in these experiments, a large decrease in the magnitude of $\Delta P$ is taken as an indicator for flooding.

The air flow rate is increased till flooding is observed. It is seen from the figure that there is an increase in the magnitude of $\Delta P$ as the air flow rate is increased to the point of flooding. This increase is found to be equal to $\rho j_{g}^{2} / 2$ which is the dynamic pressure of the upward flowing air. For the case of $j_{i}=0.63 \mathrm{~kg} / \mathrm{s}$ shown in Fig. $2, j_{g}$ was found to be around $3.8 \mathrm{~m} / \mathrm{s}$ giving a value of $0.02 \mathrm{kN} / \mathrm{m}^{2}$ which is very close to what was observed experimentally as shown in the figure. This indicates that the dynamic air pressure acts to increase the overall magnitude of the pressure gradient before and 'just before flooding'. During this period of time, droplet entrainment is observed as shown in the 'top view' flow visualization image in Fig. 3(a). The 'side view' image shows the beginning of wave formation. As the air flow rate is further increased, the flow transitions into the 'just before flooding' regime where there are intermittent peaks in the pressure signal. The flow visualization images in Fig. 3(b) also show a more disturbed liquid film and increased droplet entrainment. This is the regime where the flow starts to become chaotic yet without achieving flooding. The intermittent peaks in the pressure signal are generated in response to the flow reversals that occur intermittently at this air flow rate.

In the 'after flooding' regime, it can be seen that the $\Delta P$ time signal is unsteady. The signal can be considered to be composed of random fluctuations superimposed upon a mean value. The turbu- lent motion of the air-water mixture translating the length of the test section as a packet of fluid in the 'after flooding' regime gives rise to these random fluctuations. In this flow regime, the flow of liquid is partially reversed at regular intervals. When the liquid flow is reversed, the 'top view' flow visualization image in Fig. 3(c) shows that the cross section of the tube where the flow reversal takes place is completely bridged. Since void fraction measurements were not carried out in the present study, it was difficult to infer whether the bridge was composed entirely of liquid or a two-phase mixture. But what it does indicate is that even in large diameter vertical tubes like the one considered in the present study, there is a possibility of either the liquid or a two-phase mixture bridging the tube. In the region below this bridge, there is a temporary increase of pressure inside the test section as the flow of air is restricted temporarily. This causes an increase in the magnitude of pressure $P_{B}$ located near the air inlet. It is because of this increase in $P_{B}$, the resulting magnitude of $P_{A}-P_{B}$, i.e. $\Delta P$, decreases as observed in Fig. 2 . However, the lifespan of the bridge is short-lived as the higher momentum upward flowing jet of air penetrates this fluid and breaks it apart. Part of this fluid falls back into the test section and part of it is carried out. When the bridge breaks apart, the magnitude of pressure $P_{B}$ decreases resulting in an increase in $\Delta P$. This process of bridging and break up results in the oscillation of the pressure signal as seen in Fig. 2. The oscillation of the pressure signal continues as long as the air flow rate is greater than or equal to that required for flooding. Furthermore, Fig. 2 shows that $\Delta P$ returns to its initial value as the one before flooding when the air flow rate is decreased.

The constancy of the incoming liquid flow rate in Fig. 2 is noteworthy. It is to be noted here that with the geometric design of the test section used in these experiments, the point of liquid injection does not come into contact with the upward flowing gas. This ensures that there is a constant liquid flow into the test section regardless of the occurrence of flooding.

Fig. 4 shows the variation of the rms value of pressure gradient at flooding as a function of the liquid superficial velocity. The rms value of the pressure gradient is given by

$$
\frac{\Delta P_{r m s}}{H}=\sqrt{\frac{\sum_{i=1}^{N}\left(\Delta P_{i}-\overline{\Delta P}\right)^{2}}{N H^{2}}}
$$

Here $\Delta P_{i}$ is the instantaneous value of the differential pressure during post flooding, $\overline{\Delta P}$ is the time averaged value of differential pressure for the duration of post flooding, $N$ is the total number of samples and $H$ is the distance between the pressure ports. It can be seen from the figure that the $\Delta P_{r m s} / H$ increases linearly with the liquid flow rate. This implies that the magnitude of the low frequency oscillations that the fluid packet undergoes increases with the liquid flow rate. The increase in the magnitude of oscillations at higher liquid flow rates can be explained by comparing the fluid packet momentum to that of the jet of air forcing this packet upwards. As seen in the flow visualization images in Fig. 3, either the liquid or a two-phase mixture bridges the test section at flooding and this rising bridge of fluid eventually loses momentum. When this momentum falls below the momentum of the jet of air underneath, the fluid bridge breaks apart and part of the liquid falls back into the test section and part of it is entrained out of the test section. At higher liquid flow rates, the momentum of the liquid falling back into the test section and eventually being driven upwards is large enough to cause a large increase in the magnitude of the pressure signal oscillations as compared to lower liquid flow rates. Also superimposed in Fig. 4 is the inter-dependance of liquid flow rate and the superficial air velocity at flooding. The data is in conformity with previous research (Wallis, 1961; Dukler et al., 1984; Hewitt, 1989; Vijayan et al., 2001) indicating that the amount of air flow rate required to induce flooding is inversely proportional to the liquid flow rate. 
(a)

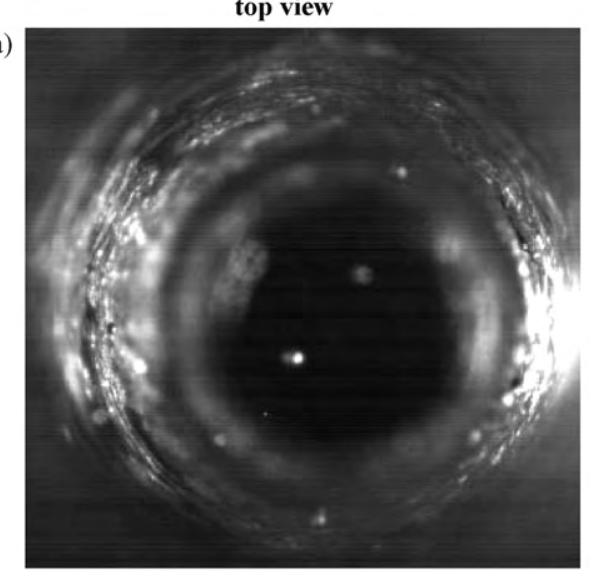

(b)

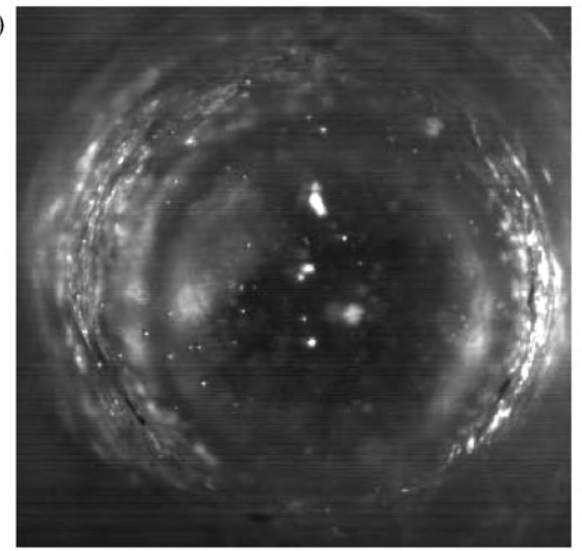

(c)

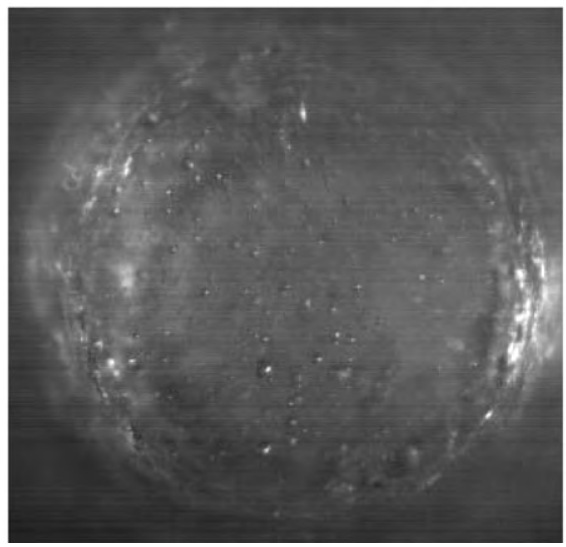

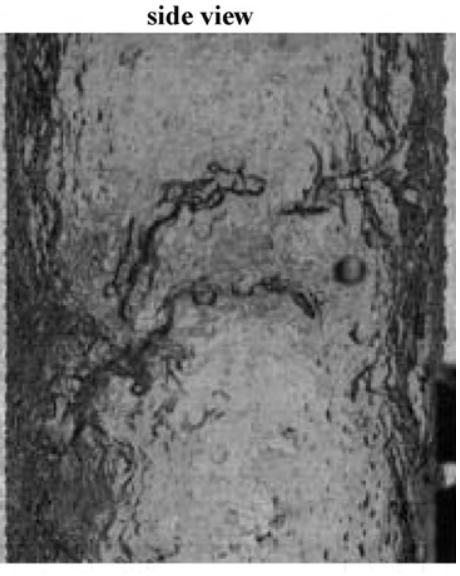
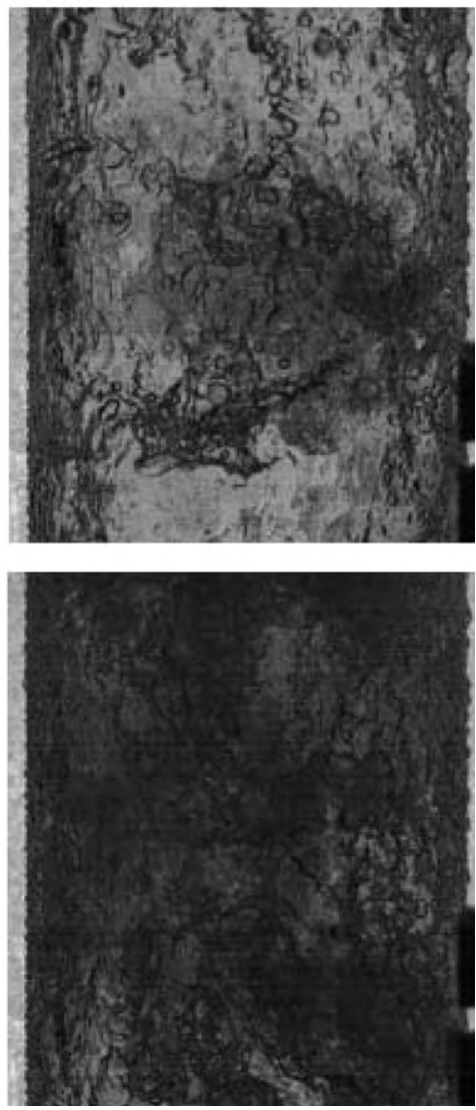

Fig. 3. Flow visualization images showing air-water flow for the following cases. (a) Droplet entrainment, (b) just before flooding and (c) after flooding.

\subsection{Mean wall pressure profiles}

The mean wall pressure profiles along the length of the test section are an indication of the propagation of the pressure disturbances in the air-water annular flow. Fig. 5 shows the mean axial wall pressure profiles measured at five different locations along the length of the test section, the first location being $0.20 \mathrm{~mm}$ from the air inlet. The profiles are measured for three flow conditions, namely 'no air flow', 'just before flooding' and 'after flooding'. 'No air flow' corresponds to the condition where there is only annular liquid flow through the test section. The 'just before flooding' condition corresponds to that where there is large scale liquid droplet entrainment but no flow reversal of any scale. 'After flooding' corresponds to that condition where there is partial or complete flow reversal at regular intervals. It can be seen from the figure that for all the three flow rates considered in the study, there is a general decrease in the wall pressure as one moves away from the water inlet and towards the air inlet. The decrease in the wall pressure is due to flow acceleration of the falling liquid film. As mentioned in the previous section, flow acceleration results as the falling annular film is not fully developed. However, the decrease in wall pressure is nearly the same for the conditions of 'no air flow' and 'just before flooding' for all the three cases. A maximum wall pressure of about $1.0 \mathrm{kN} / \mathrm{m}^{2}$ was found to be at the location closest to the water inlet.

It can also be seen from Fig. 5 that the pressure curve for the 'just before flooding' case shifts upwards indicating that there is a marginal increase in the magnitude of wall pressures at all locations as compared to the 'no air flow' case. This implies that the amount of air flow that causes droplet entrainment in the test section is not large enough to cause any drastic changes in the wall pressures. At this flow condition, the upward flowing air causes the formation of waves in the liquid film. Liquid droplets are sheared 


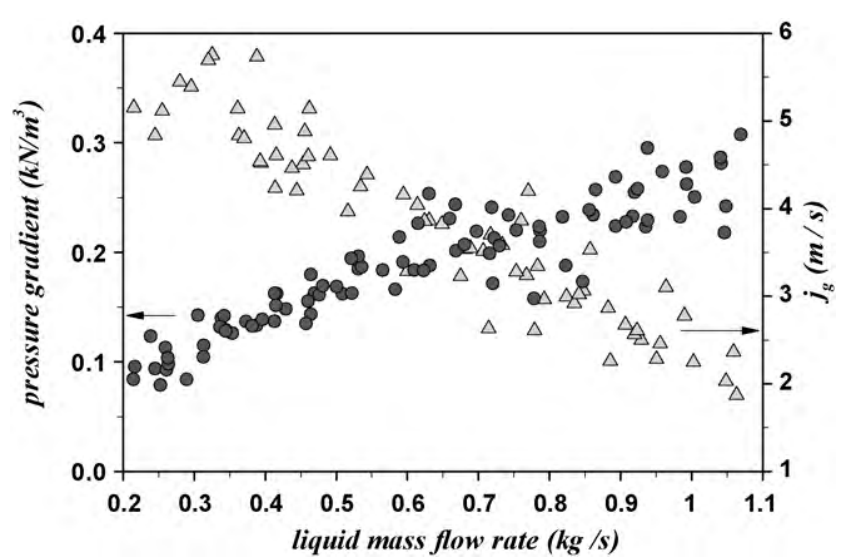

Fig. 4. Variation of axial pressure gradient and superficial gas velocity with liquid mass flow rate for the 'after flooding' case.
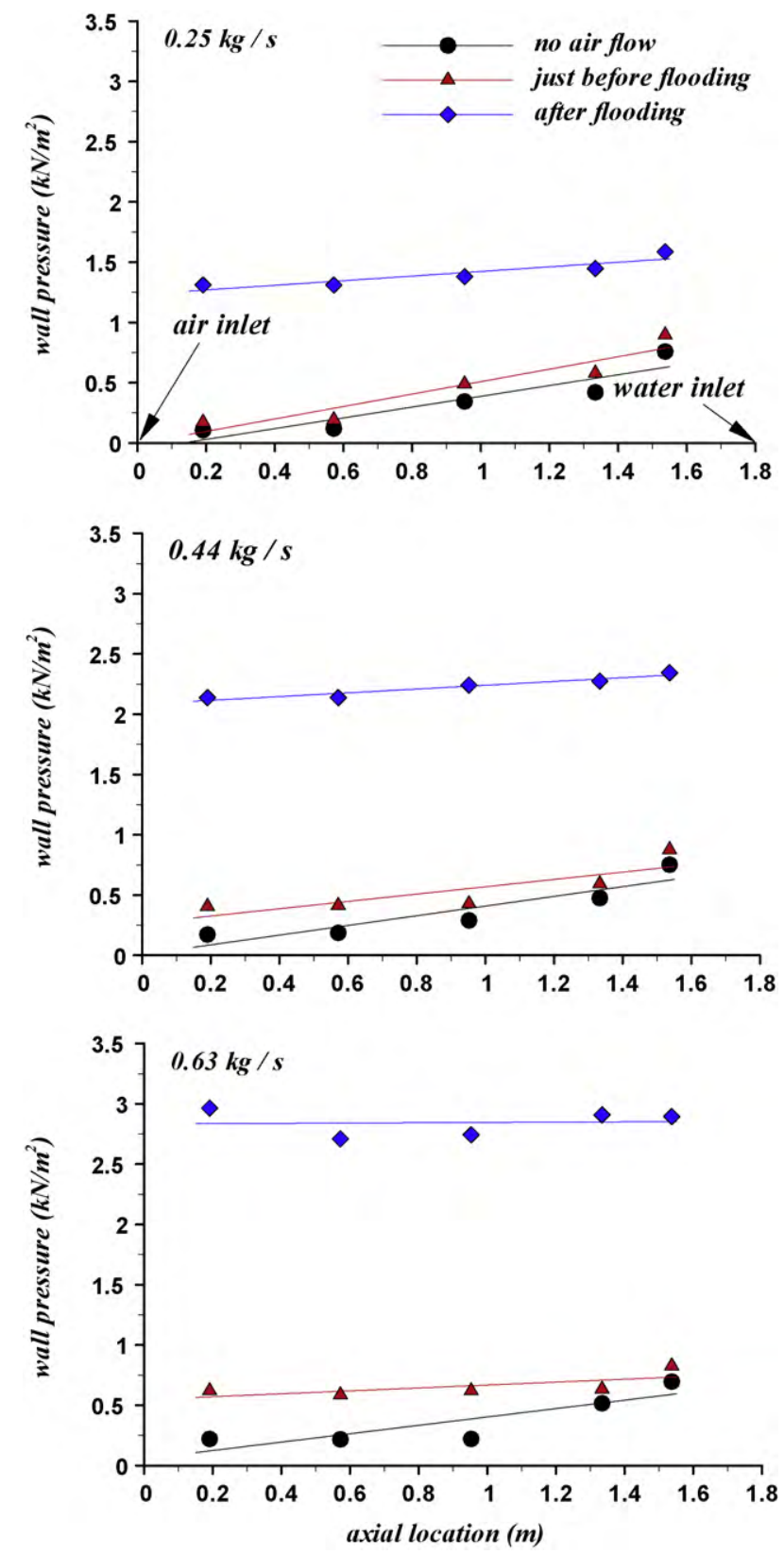

Fig. 5. Mean axial wall pressure profiles at different liquid flow rates. off from the interface and are deposited downstream on the liquid film. Because of this deposition, there is a marginal increase in the momentum of the air-water mixture. It is this marginal increase in momentum that causes the marginal increase in wall pressure for the 'just before flooding' case. However, it can be seen for the 'after flooding' case the pressure curve shifts further upwards indicating that there is large increase in the wall pressures at all locations. This implies that the wall pressures are only affected when there is maximum interfacial distortion. Under these conditions, a highly turbulent two-phase mixture is observed along with repeated formation and break up of slugs of fluid that traverse the length of the test section. The formation and convection of these slugs of fluid greatly increases the momentum that eventually results in a large increase in the wall pressures. This can be explained by considering the Poisson equation for turbulent flow which can be written as follows:

$-\frac{1}{\rho} \frac{\partial^{2} p}{\partial x^{2}}=\frac{\partial u}{\partial y} \frac{\partial v}{\partial x}$

Here, the pressure $P$ and the axial and radial velocities $u$ and $v$ are comprised of both the mean and fluctuating components. The right hand side of Eq. (7) is the forcing function that primarily arises due to the momentum of the flow. In a highly turbulent flow like the 'after flooding' case, the increase in momentum comes from eddy collisions that greatly increases the rate of strain as well as eddy rotation that generates vorticity (Bradshaw and Koh, 1981). An increase in momentum due to the generation of vorticity and increased rate of strain increases both the mean and fluctuating components of pressure as observed for the 'after flooding' case in Fig. 5.

Fig. 6 shows the comparison of the mean wall pressure profiles at different liquid flow rates for the three conditions of 'no air flow', 'just before flooding' and 'after flooding'. It can be seen that for the 'no air flow' and 'just before flooding' cases, the mean wall pressure at the location closest to the water inlet, i.e. at $1.54 \mathrm{~m}$ from the air inlet, is the same for all the three liquid flow rates. Even though there is a little variation in the wall pressure magnitudes at other locations, the wall pressures seem to be independent of the air flow rate at location $1.54 \mathrm{~m}$ for any given liquid flow rate for the cases of 'no air flow' and 'just before flooding'. This shows that the pressure disturbances are damped out by the time they reach this location. Hence, the effect of air flow rate for any given liquid flow rate is least seen at this location for the cases of 'no air flow' and 'just before flooding'. For the 'after flooding' case, it can be seen from Fig. 6 that there is considerable change in the wall pressure magnitude at all the axial locations for the three different liquid flow rates. This suggests that a large change in the test section pressure for countercurrent annular flow in large diameter vertical tubes only occurs after flooding is initiated and only when there is low frequency oscillation of the fluid packet as observed in Fig. 2. The interfacial pressure disturbances produced just before flooding' are observed not to be strong enough in magnitude to produce any kind of mean wall pressure change upstream of the air inlet.

The constancy of wall pressures at location $1.54 \mathrm{~m}$, i.e. at the location farthest from the air inlet (Fig. 6a and b) further suggests that incipient flooding starts at a location that is closer to the air inlet for countercurrent annular flows in large diameter vertical tubes. To confirm this, preliminary flow visualization test were carried out using the setup described in Section 2.2. Incipient flooding location is the axial location along the test section where the initial flow reversal is observed. The location of incipient flooding is the starting point for the growth and propagation of waves that lead to flooding. In an effort to qualitatively determine this location in the present experiments and confirm the observations made through mean wall pressure measurements, the flow visualization images were analyzed. Fig. 7 shows the flow visualization of the bottom 

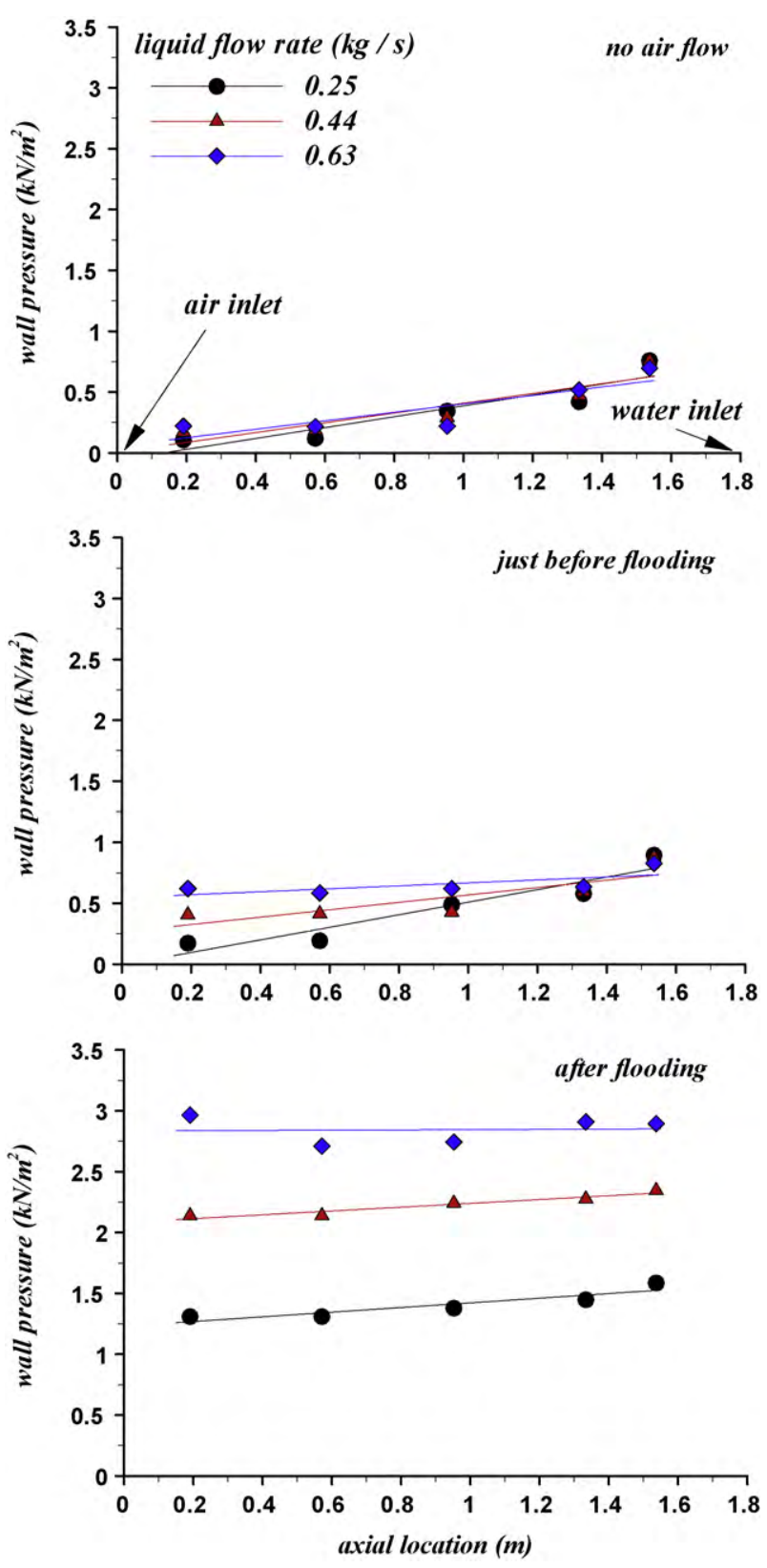

Fig. 6. Mean axial wall pressure profiles for the three different flow conditions. part of the test section and a schematic showing the spreading of the air jet inside the test section. From the flow visualization images, the location $L_{a}$ where waves began to first appear after introducing the air flow was determined to be $375 \mathrm{~mm}$ downstream of the air inlet tube. It can be said that the flooding instability is initiated at this location and the waves first begin to appear at this location and consequently propagate upstream. The waves are carried upstream due to the action of the upward flowing jet of air and may or may not grow in amplitude as will be explained in the following section. The location where the jet boundary comes into contact with the falling liquid film is dependent on the air inlet tube diameter and the spreading of the jet. As explained in the following paragraph, for a larger diameter inlet tube, this location would be closer to the air inlet and for a smaller diameter tube, it would be farther away from the air inlet.

The diameter of the air inlet tube changes the location at which the outer boundary of the jet of air comes into contact with the falling liquid film. As shown in Fig. 8, a jet of air from a smaller diameter tube travels a larger distance as compared to a jet of air from a larger diameter tube before the outer jet boundary comes into contact with the falling liquid film. As seen from Fig. 8, this is due to the fact that the rate at which jet spreading occurs is constant regardless of the diameter. The rate of air jet spreading for a jet of diameter $d$ is given in terms of its half-width. The half-width $y_{1 / 2}$ of a jet corresponds to the width of a jet at an axial location $x$ where the axial velocity is half of the jet centerline velocity at that location. The half-width of a jet is given by $y_{1 / 2}=K x$. Here $K$ is a constant whose value is about 0.1 for a round jet (Wygnanski and Fiedler, 1969). From this equation, it can be seen that the slope of the above equation given by $d y_{1 / 2} / d x$ is constant regardless of the diameter, i.e. the spreading rate is constant regardless of the diameter of the air inlet tube. However, the point of contact of the air jet with the falling liquid film changes as the inlet tube diameter is changed. This point of contact can be considered as the location where the initial flow reversal takes place. As seen from Fig. 8, this point of contact with the falling liquid film is largely determined by the spreading rate of the air jet which in turn influences the flooding behavior. It also indicates that the initial instability and hence the initial flow reversal due to the interaction of the air jet and the liquid film primarily occurs near the water outlet and in fact moves closer to the water outlet as the diameter of the air inlet tube is increased.

Furthermore, it should also be noted that since the distance traveled by the jet of air before coming into contact with the falling liquid film is greater for a smaller diameter air inlet tube as compared to a larger diameter tube, the decay in the velocity or the loss in momentum of the air jet from the smaller diameter tube is greater. This implies that greater air velocities are required to induce flooding with smaller diameter air inlet tubes as compared to larger diameter air inlet tubes for a given mass flow rate.

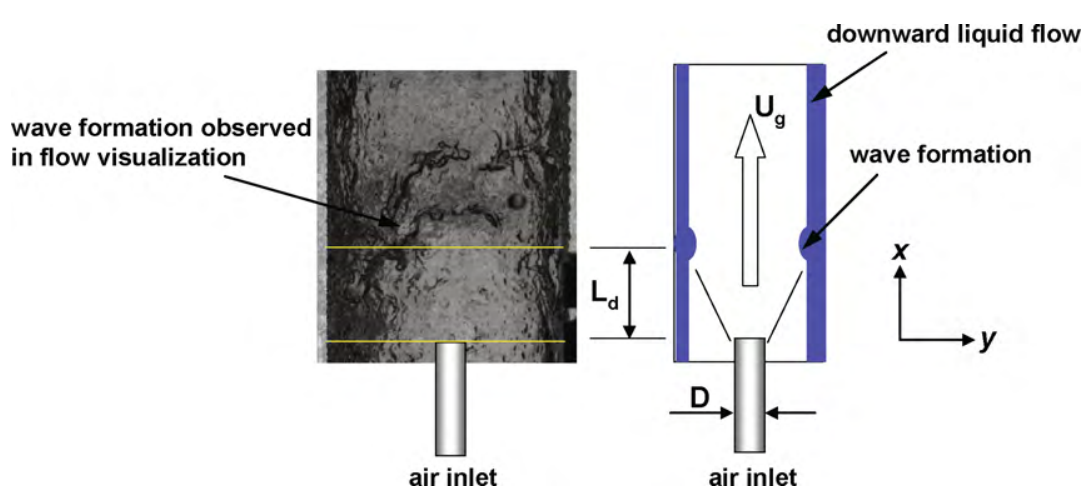

Fig. 7. Flow visualization image and a corresponding schematic illustrating the location of incipient flooding. 


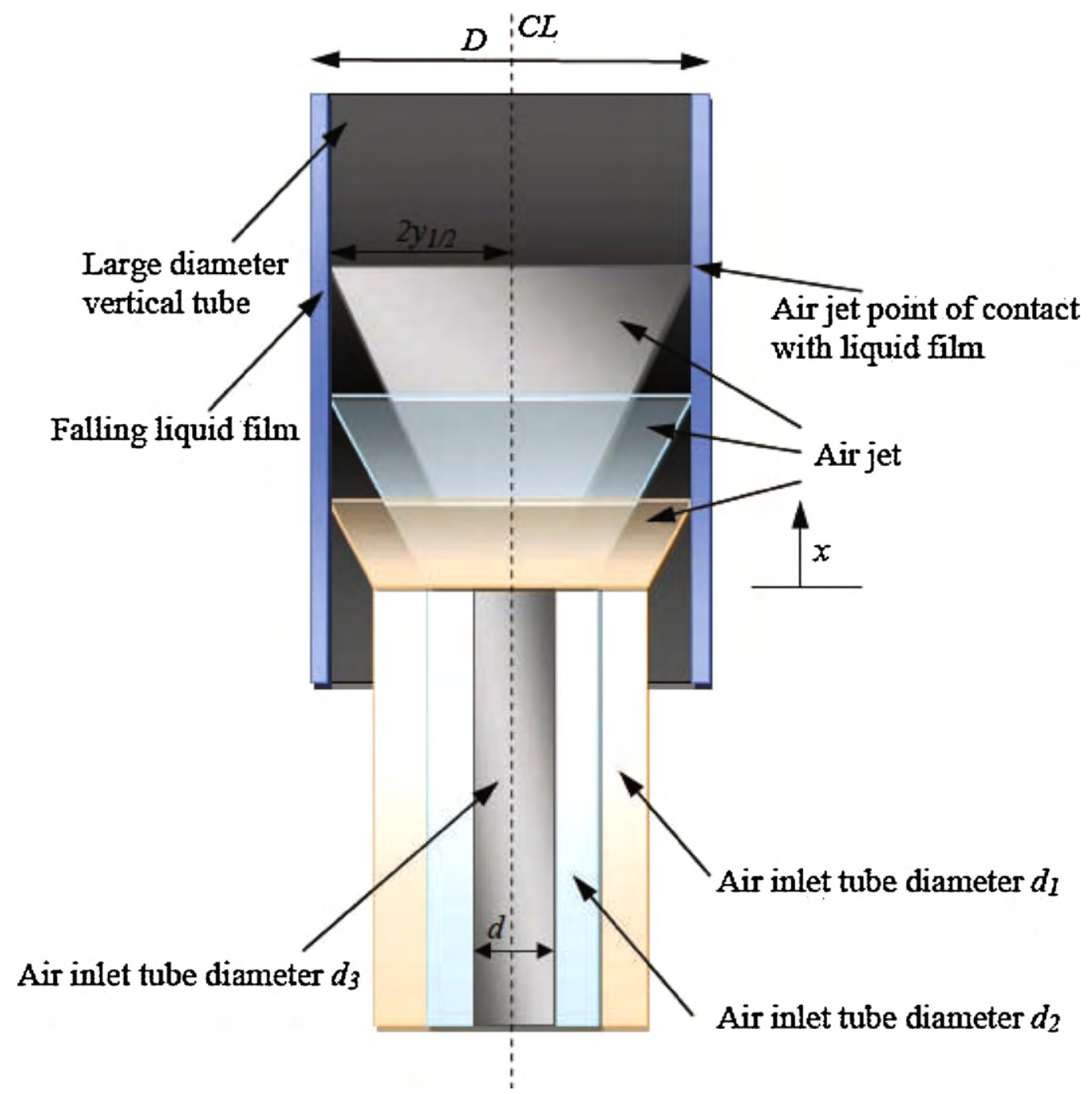

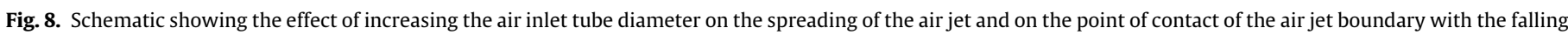
liquid film; $D 1>D 2>D 3$.

\subsection{Wall pressure frequency spectra}

Fig. 9(a) shows the time variation of the Kulite transducer signal for the three cases of 'no air flow', 'just before flooding' and 'after flooding' for a liquid flow rate of $0.44 \mathrm{~kg} / \mathrm{s}$. It can be seen from the figure that the pressure signal for the 'just before flooding' case has a higher magnitude and is comparatively more unsteady than the 'no air flow' case. The unsteadiness in the signal is due to pressure disturbances that propagate through the film and reach the transducer location. The pressure disturbances can arise from a number of sources such as interfacial disturbances and also convected pressure disturbances due to the entrained liquid droplets in the test section (Samways et al., 1997). The figure further shows that maximum signal unsteadiness is only observed in the 'after flooding' case. In order to derive the frequencies present in the flow, a discrete fast fourier analysis was carried out on the Kulite pressure signals in Fig. 9(a) and the corresponding frequency spectra is shown in Fig. 9(b). As expected, the results show that for the
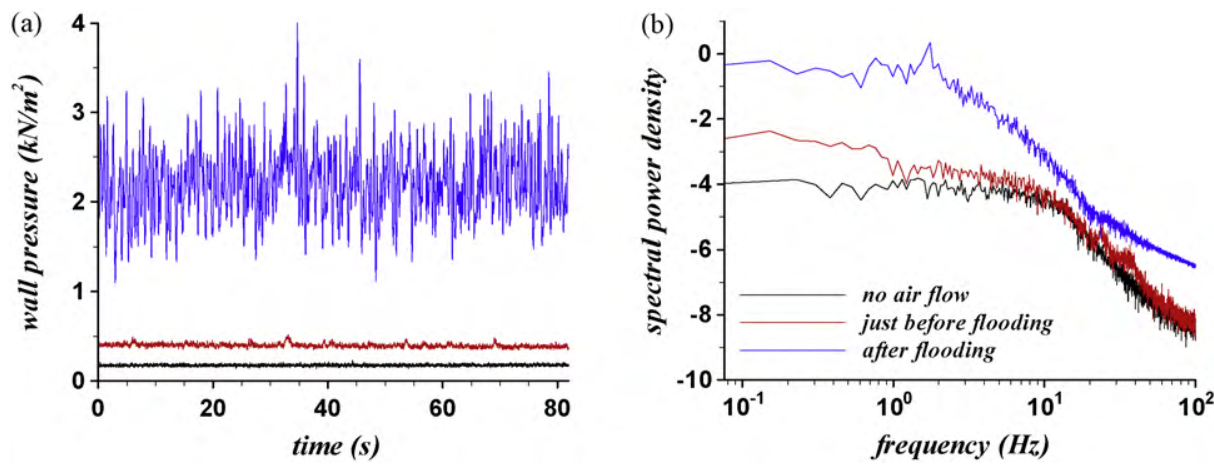

Fig. 9. (a) Time variation of the Kulite pressure transducer signal for the three cases mentioned in (b); (b) frequency spectra of pressure signals corresponding to (a). 

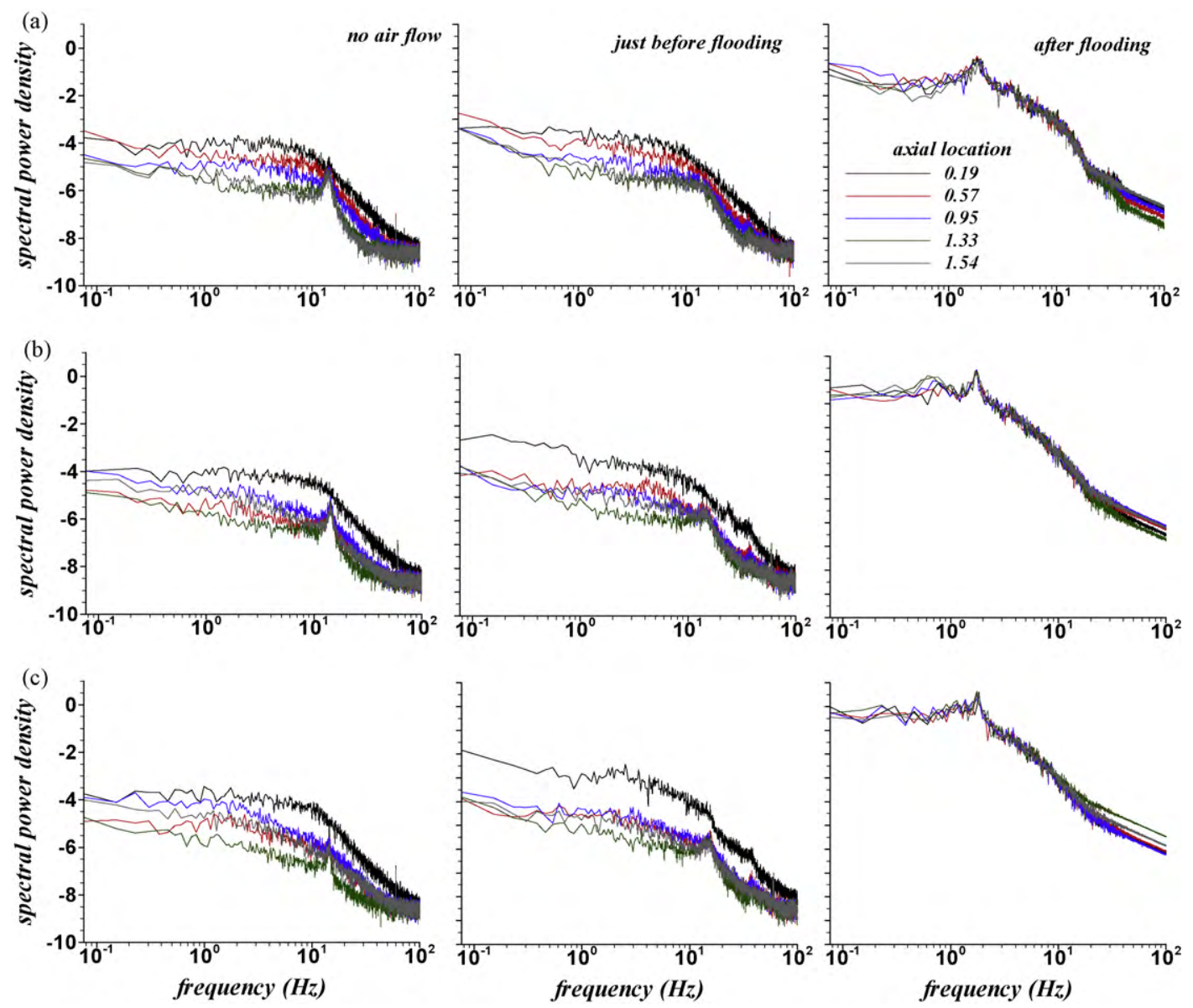

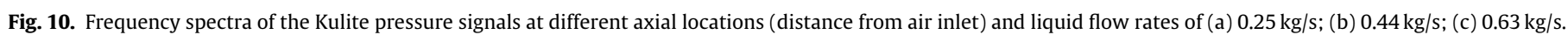

'no air flow' case, there is no single dominant frequency present in the flow. Surprisingly, for the 'just before flooding' case, it is seen that there is no dominant frequency in the flow as well. The figure shows that there is only a continuous band of frequencies present for both the cases. For the 'after flooding' case, it is seen from the figure that there is a distinct dominant low frequency phenomenon taking place at about $1.73 \mathrm{~Hz}$. Furthermore, the amplitude of the entire frequency spectrum for the 'after flooding' in terms of the spectral power density is much higher than the other two cases as shown in the figure.

In order to understand the results more thoroughly, the frequency spectra for the conditions of 'no air flow', 'just before flooding' and 'after flooding' were compared with each other for different flow conditions as shown in Fig. 10. It can be seen from the figure that for the 'no air flow' and 'just before flooding' cases, the magnitude of the spectral power density increases as the axial location moves closer to the water inlet of the test section. However, as observed before there is no single dominant frequency present in the flow and there is continuous spectrum of frequencies present. The absence of a single dominant frequency, especially for the 'just before flooding' case shows that there is a combination of waves with different wavelengths that are collectively responsible for flooding as opposed to a single wave. The data further shows that for the 'after flooding' case, there is a dominant low frequency tone with a frequency of $1.73 \mathrm{~Hz}$. A close inspection of flow visualization results have shown that this frequency corresponds to the low frequency oscillation of the fluid packet that forms after flooding. It is interesting to note that there is no variation in this frequency for the different flow rates considered in this study. It is further seen that no given frequency is amplified but there is rather an overall increase in the magnitude of the spectral power density for all the frequencies. This further confirms that a single disturbance wave may not be responsible for flooding in large diameter vertical tubes. Furthermore, the spectra at all the axial locations for a given flow rate collapse into one for the 'after flooding' case as seen in the figure. This implies that the same frequencies simultaneously occur at different axial locations within the test section indicating the presence of a highly turbulent liquid film that is characteristic of flooding.

\section{Conclusions}

The characteristics of countercurrent annular air-water flow in a large diameter vertical tube have been examined up to and after flooding using wall pressure measurements for three liquid flow rates of $0.25,0.44$ and $0.63 \mathrm{~kg} / \mathrm{s}$. Mean pressure profiles along the length of the test section were measured using fast response Kulite pressure transducers. The results show that the magnitude of mean wall pressure increased downstream of the air inlet toward the water inlet for all the flow conditions considered in the study. The results further showed that the magnitude of mean wall pressure at the location closest to the water inlet is independent of the flow conditions up to flooding suggesting that flooding is initiated in the lower part of the test section near the air inlet. Fourier transform of the pressure signals showed that there is a wide spectrum of frequencies for waves at the air-water interface that exist up to flooding. Since no single dominant frequency was observed in the Fourier spectra at any of the axial locations, the data implies that flooding 
may be caused due to the constructive superposition of a plurality of waves rather than the action of a single large-amplitude wave.

\section{Acknowledgements}

The authors are thankful to Mr. Matt Solmos and Mr. Peter Maginot for their help in setting up the experimental facility. The financial support of U.S. DoE and Nuclear Engineering Education Research Program under award number DE-FG07-05ID14696 is gratefully acknowledged.

\section{References}

Bankoff, S.G., Lee, S.C., 1986. A critical review of the flooding literature. In: Hewitt, G.F., Delhaye, J.M., Zuber, N. (Eds.). Hemisphere. Multiphase Science and Technology.

Bradshaw, P., Koh, Y.M., 1981. A note on Poisson's equation for pressure in a turbulent flow. Phys. Fluids 24 (4), 777.

Cai, Y., Wambsganss, M.W., Jendrejczyk, J.A., 1996. Application of Chaos theory and identification of two-phase flow patterns and transitions in a small, horizontal, rectangular channel. ASME J. Fluids Eng. 118, 383-390.

Davis, M.R., 1973. Pressure fluctuations in a vapour-liquid mixture flow. Int. J. Heat Mass Transfer 16, 2043.

Drahos, J., Tihon, J., Serio, C., Lubbert, A., 1996. Deterministic Chaos analysis of pressure fluctuations in a horizontal pipe at intermittent flow regime. Chem. Eng. J. 64, 149-156.

Dukler, A.E., Smith, L., Chopra, A., 1984. Flooding and upward film flow in tubes. I. Experimental studies. Int. J. Multiphase Flow 10, 585-597.

Hewitt, G.F., 1989. Countercurrent two-phase flow. In: Proc. NURETH-4 (4th Int. Top. Mtg on Nuclear Reaction Thermal Hydraulics, pp. 1129-1144.
Hubbard, M.G., Dukler, A.E., 1966. The characterization of flow regimes for horizontal two-phase flow. I. Statistical analysis of wall pressure fluctuations. In: Proceedings of the Heat Transfer and Fluid Mechanics Institute, Santa Clara, CA, June, pp. 100-121.

Langford, H.M., Beasley, D.E., Ochterbeck, J.M., 1998. Observations on Chaos in upward gas-liquid flow. In: Proceedings of the ASME International Mechanical Engineering Conference and Exposition, HTD 361-5, pp. 247-254.

Matsui, G., 1984. Identification of flow regimes in vertical gas-liquid two-phase flow using differential pressure fluctuations. Int. J. Multiphase Flow 10, 711-720.

McClusky, H.L., Holloway, M.V., Beasley, D.E., Ochterbeck, J.M., 2002. Continuous wavelet transforms of instantaneous wall pressure in slug and churn upward gas-liquid flow. J. Fluids Eng. 124, 625-633.

Nishikawa, K., Sekoguchi, K., Fukano, T., 1969. On the pulsation phenomena in gas-liquid two-phase flow. Bull. JSME 12, 1410-1416.

Sami, S.M., Lakis, A.A., 1986. Spectral analysis of wall-pressure fluctuations in turbulent two-phase flow. J. Acoust. Soc. Am. 80 (5), 1392-1403.

Samways, A.L., Bradbury, L.J.S., Bruun, H.H., 1997. Pressure measurements and convection velocity evaluations in two-phase flow. Int. J. Multiphase Flow 23 (6), 1007-1029.

Semenov, N.I., 1959. Pressure pulsations during the flow of gas-liquid mixtures in Pipes, United States Atomic Energy Commission, Tech. Report 4496.

Solmos, M.A., 2008. An experimental investigation of the countercurrent flow limitation. M.S. Thesis, Texas A\&M University, College Station, TX.

Tutu, N.K., 1982. Pressure fluctuations and flow pattern recognilion in vertical twophase gas-liquid flows. Int. J. Multiphase Flow 8, 443-447.

Vijayan, M., Jayanti, S., Balakrishnan, A.R., 2001. Effect of tube diameter on flooding. Int. J. Multiphase Flow 27, 797-816.

Wallis, G.B., 1961. Flooding velocities for air and water in vertical tubes. Technica report, AEEW-R-123, United Kingdom Atomic Energy Authority. Reactor Group. Atomic Energy Establishment, Winfrith, Dorset.

Wygnanski, I., Fiedler, H., 1969. Some measurements in the self-preserving jet. J. Fluid Mech. 38, 577-612. 